\title{
Educação em saúde no aconselhamento contraceptivo para esterilização cirúrgica
}

\author{
Health education in the contraceptive counseling for surgical sterilization
}

\section{Educación sanitaria en el asesoramiento anticonceptivo para esterilización quirúrgica}

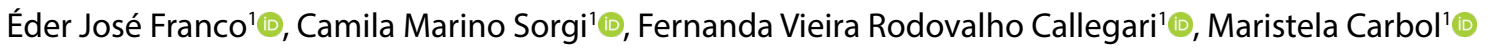 \\ ${ }^{1}$ Universidade Federal de São Carlos (UFSCar), São Carlos, São Paulo, Brasil
}

\section{Resumo}

Introdução: O planejamento reprodutivo deve levar em conta as condições de vidas das pessoas e garantir que possam decidir sobre sua reprodução de forma livre e esclarecida. Objetivo: Avaliar a atividade de educação em saúde no processo de aconselhamento contraceptivo para esterilização cirúrgica. Metódos: Trata-se de um estudo observacional descritivo, tipo inquérito. A pesquisa ocorreu no Centro Municipal de Especialidades Médicas da cidade de São Carlos, estado de São Paulo, com a participação dos usuários da atenção básica que manifestaram desejo pela esterilização cirúrgica e compareceram na atividade de educação em saúde, no período de setembro a dezembro de 2016. A coleta de dados foi por meio de questionário estruturado aplicado pelos pesquisadores ao término das atividades de educação em saúde. As respostas das questões foram armazenadas no programa Microsoft Excel 2010 para calcular frequências absolutas, relativas e médias. A análise dos dados quantitativos permitiu a interpretação descritiva das informações. Resultados: Foram realizadas seis atividades de educação em saúde com participação de 45 indivíduos, 26 mulheres (58\%) e 19 homens (42\%). A idade média das mulheres e dos homens foi 31,4 e 37,5 anos, respectivamente. A maioria tinha dois filhos vivos com o atual parceiro. Os motivos alegados para esterilização cirúrgica foram número suficiente de filhos, questões financeiras desfavoráveis e problemas de saúde da mulher. As dúvidas sobre métodos contraceptivos e procedimentos cirúrgicos foram problematizadas durante educação em saúde e nenhuma nova dúvida surgiu quando os participantes foram entrevistados. Após a educação em saúde, dois casais mostraram-se interessados pela mudança da laqueadura tubária para vasectomia. Chamou à atenção de duas mulheres o dispositivo intrauterino e o contraceptivo hormonal transdérmico, mas mantiveram a escolha pelo método definitivo. Conclusão: As atividades de educação em saúde permitiram aos usuários esclarecimentos de dúvidas sobre a esterilização cirúrgica e reflexão sobre a possibilidade de mudanças para outros métodos contraceptivos.

Palavras-chave: Educação em Saúde; Aconselhamento; Esterilização Reprodutiva.
Como citar: Franco EJ, Sorgi CM, Callegari FVR, Carbol M. Educação em saúde no aconselhamento contraceptivo para esterilização cirúrgica. Rev Bras Med Fam Comunidade. 2020;15(42):2082. https://doi.org/10.5712/rbmfc15(42)2082
Autor correspondente:

Éder José Franco.

E-mail: ederjosefranco@hotmail.com

Fonte de financiamento:

Programa Institucional de Bolsas de Iniciação Científica - PIBIC/CNPq/UFSCar. Parecer CEP: aprovado.

Número do parecer: 1.683.384.

Procedência e revisão por pares: revisado por pares.

Recebido em: 16/05/2019.

Aprovado em: 01/11/2019. 


\begin{abstract}
Introduction: Reproductive planning must take into account people's living conditions and ensure that they can decide on their reproduction in a free and informed manner. Objective: To evaluate the health education activity in the contraceptive counseling process for surgical sterilization. Methods: This is a descriptive observational survey study. The research took place at the Municipal Center of Medical Specialties of the city of São Carlos, state of São Paulo, with the participation of primary care users who expressed a desire for surgical sterilization and attended the health education activity, from September to December 2016. Data collection it was through a structured questionnaire applied by researchers at the end of health education activities. The answers to the questions were stored in the Microsoft Excel 2010 program to calculate absolute, relative and average frequencies. The analysis of quantitative data allowed the descriptive interpretation of the information. Results: Six health education activities were conducted with the participation of 45 individuals, 26 women (58\%) and 19 men (42\%). The average age of women and men was 31.4 and 37.5 years, respectively. Most had two children alive with the current partner. Alleged reasons for surgical sterilization were sufficient numbers of children, unfavorable financial issues, and women's health problems. Doubts about contraceptive methods and surgical procedures were problematized during health education and no new questions arose when participants were interviewed. After health education, two couples were interested in switching from tubal ligation to vasectomy. Two women caught the attention of the intrauterine device and the transdermal hormonal contraceptive, but maintained the choice of the definitive method. Conclusion: Health education activities allowed users to clarify doubts about surgical sterilization and reflect on the possibility of changes to other contraceptive methods.
\end{abstract}

Keywords: Health Education; Counseling; Reproductive Sterilization.

\title{
Resumen
}

Introdutión: La planificación reproductiva debe llevar en cuenta las condiciones de vida de las personas y garantizar que puedan decidir sobre su reproducción de forma libre y clara. Objetivo: Evaluar la actividad de la educación en salud en el proceso de asesoramiento contraceptivo para la esterilización quirúrgica. Método: Este es un estudio descriptivo de encuesta observacional. La investigación tuvo lugar en el Centro Municipal de Especialidades Médicas de la ciudad de São Carlos, estado de São Paulo, con la participación de usuarios de atención básica que expresaron su deseo de esterilización quirúrgica y asistieron a la actividad de educación sanitaria, de septiembre a diciembre de 2016. Recopilación de datos a través de un cuestionario estructurado aplicado por investigadores al final de las actividades de educación sanitaria. Las respuestas a las preguntas se almacenaron en el programa Microsoft Excel 2010 para calcular las frecuencias absolutas, relativas y medias. El análisis de datos cuantitativos permitió la interpretación descriptiva de la información. Resultados: Fueron realizadas seis actividades de educación en salud con 45 individuos, 26 del sexo femenino (58\%) y 19 masculino (42\%). La edad media de ambos sexos fue de 31,4 y 37,5 años respectivamente. La mayoría tenía dos hijos vivos con el actual compañero. Los motivos alegados para la esterilización quirúrgica fueron el número suficiente de hijos, dificultades financieras y problemas de salud de la mujer. Las dudas sobre los métodos contraceptivos y procedimientos quirúrgicos fueron problematizadas durante la educación sanitaria y ninguna duda surgió cuando se entrevistó los participantes. Después de la educación en salud, dos parejas se mostraron interesados por cambiar la ligadura de trompas para vasectomía. Dos mujeres se interesaron por el dispositivo intrauterino y el contraceptivo hormonal transdérmico, pero al final mantuvieron la opción por el método definitivo. Conclusión: Las actividades de educación sanitaria permitieron a los usuarios esclarecimiento de dudas sobre la esterilización quirúrgica y reflexión sobre las posibilidades de cambios para otros métodos anticonceptivos.

Palabras clave: Educación Sanitaria; Asesoramiento; Esterilización Reproductiva.

\section{INTRODUÇÃO}

No Brasil, por um longo tempo as mulheres lutaram para que as medidas de planejamento familiar fossem incluídas às políticas de saúde, pois no começo do século XX os programas de saúde da mulher eram direcionados aos cuidados na gestação e no parto. Somente em 1983 houve a implantação dos serviços de planejamento familiar com a criação do Programa de Assistência Integral à Saúde da Mulher (PAISM) pelo Ministério da Saúde, constituindo-se uma referência histórica no país, pois incluiu estratégias de acesso à população aos meios de contracepção. ${ }^{1}$

O planejamento familiar trouxe à tona discussões sobre os direitos sexuais e reprodutivos, desvinculando a maternidade da vida sexual, reconhecendo que todo cidadão tem o direito de receber informações e meios que permitam planejar o momento oportuno para ter seus filhos e liberdade de decisão sobre sua vida reprodutiva. ${ }^{2,3}$ Desta forma, o PAISM se antecipou à Constituição de 1988, quando alegou o direito de livre escolha das pessoas em relação à saúde reprodutiva como prevê o Art. 226 , $\S 7^{\circ}$ regulamentado pela Lei $9.263 / 1996 .{ }^{4}$ 
De acordo com a Lei da Esterilização Cirúrgica $n^{\circ}$ 9.263, de 12 de janeiro de $1996^{5}$, planejamento familiar é compreendido como:

Art. $2^{\circ}$ Conjunto de ações de regulação da fecundidade que garanta direitos iguais de constituição, limitação ou aumento da prole pela mulher, pelo homem ou pelo casal.

Para fins desta Lei, o art. $9^{\circ}$ define que:

Art. $9^{\circ}$ Para o exercício do direito ao planejamento familiar são oferecidos todos os métodos e técnicas de concepção e contracepção que sejam cientificamente aceitos e que não coloquem em risco à vida e à saúde das pessoas, sempre garantindo a liberdade de escolha.

O planejamento familiar tornou-se uma medida prioritária do Ministério da Saúde, onde todos os níveis de complexidade do Sistema Único de Saúde devem assegurar assistência à concepção e à contracepção de forma integral oferecendo aconselhamento contraceptivo às pessoas. ${ }^{6,7}$

No planejamento familiar devem-se levar em conta as condições de vida das pessoas e garantir que elas possam decidir sobre sua reprodução sem segregação, constrangimento ou repressão e hostilidade. ${ }^{8}$ No entanto, as ações voltadas à saúde reprodutiva sempre estiveram mais focadas às mulheres adultas, discriminando os adolescentes e a população masculina. ${ }^{9,10}$

Neste sentido, o termo planejamento reprodutivo passou a ser considerado mais adequado do que planejamento familiar por se tratar de uma concepção mais abrangente, uma vez que pode ser realizado por adolescentes, jovens ou adultos, homem ou mulher, individualmente ou com seu parceiro, independente de ter uma união estável. ${ }^{11}$

Para consubstanciar as ações de planejamento reprodutivo, a gestão municipal deve garantir recursos adequados ao funcionamento das unidades de saúde da atenção básica, provendo-se de meios materiais, inovações e dispositivos suficientes para desenvolver o aconselhamento contraceptivo; além de ter uma rede de referência estruturada., ${ }^{9,12}$

Para as pessoas planejarem a vida conforme suas necessidades e para uma prática sexual mais saudável, os serviços de saúde devem oferecer educação em saúde individual, ao casal e em grupo, disponibilizando informações e meios adequados para prevenir a gravidez sem colocar a vida e a saúde das pessoas em risco e de forma que elas possam escolher livremente a sua contracepção.

Os profissionais de saúde durante as atividades de educação em saúde devem aconselhar e promover a reflexão do indivíduo ou casal sobre todas as opções contraceptivas reversíveis e irreversíveis disponíveis, no que se refere à eficácia, aos efeitos colaterais, às vantagens e desvantagens. E ainda, oferecer informações sobre as técnicas cirúrgicas para realização da laqueadura tubária e vasectomia, os possíveis riscos da anestesia e da cirurgia propriamente dita, os possíveis efeitos colaterais, eficácia e dificuldade de reversão. ${ }^{13,14}$

De fato, receber o máximo de informações sobre os métodos contraceptivos, questionar, esclarecer todas as dúvidas é extremamente relevante para as pessoas que desejam um método definitivo, uma vez que, de acordo com Souza et al., ${ }^{15} 50 \%$ das mulheres esterilizadas referiram que não tinham recebido qualquer tipo de informação sobre a laqueadura tubária na época da realização da cirurgia. 
Segundo Vieira e Souza, ${ }^{16}$ ao entrevistar homens e mulheres que tinham sido esterilizados há menos de cinco anos, um quarto deles relatou que consideravam que o procedimento poderia ser facilmente reversível, demonstrando opiniões equivocadas sobre o método.

Quando a decisão por um método definitivo é baseada na compreensão dos indivíduos acerca dos métodos contraceptivos, em experiências prévias e no seu contexto de vida, considerando o número de filhos idealmente planejado ou, por ventura, doenças que podem comprometer à sua saúde, as taxas de arrependimento futuro são bem mais baixas. ${ }^{17,18}$

Isto tudo reforça o quanto a educação em saúde pode estimular a busca de soluções, contribuir para o desenvolvimento da consciência crítica das pessoas e propiciar maior autonomia para decidir sobre sua saúde e, por sua vez, melhorar a qualidade de vida. ${ }^{19}$

Neste sentido o objetivo desta pesquisa foi avaliar a atividade de educação em saúde, uma das ações do processo de aconselhamento contraceptivo para esterilização cirúrgica.

\section{MÉTODOS}

Trata-se de um estudo observacional descritivo, tipo inquérito, aprovado pelo Comitê de Ética em Pesquisa em Seres Humanos da Universidade Federal de São Carlos com número de parecer 1.683.384 e CAAE 57577516.7.0000.5504 na data de 17/08/2016.

Os usuários interessados pela esterilização cirúrgica devem passar por um aconselhamento contraceptivo multiprofissional, a fim de auxiliá-los na decisão de manter a contracepção definitiva ou optar por um método reversível, como previsto na Lei da Esterilização Cirúrgica. ${ }^{5}$ Este aconselhamento é composto por várias ações sendo elas, a atividade de educação em saúde e as avaliações clínica, psicológica e social.

Esta pesquisa teve como público-alvo os usuários da atenção básica que manifestaram desejo pela esterilização cirúrgica e participaram da atividade de educação em saúde, no período de setembro a dezembro de 2016.

As atividades de educação em saúde aconteceram no Centro Municipal de Especialidades Médicas da cidade de São Carlos, estado de São Paulo. Cada uma delas teve duração de três horas e foi realizada por meio de roda de conversa facilitada por uma enfermeira, com um público em torno de 10 pessoas.

A condução do grupo começa pela apresentação dos usuários e pelos motivos deles estarem na atividade, de modo a quebrar o gelo e predispor vinculação. Posteriormente, os métodos contraceptivos reversíveis e irreversíveis são apresentados, disparando uma problematização sobre as diferentes formas de prevenção de gravidez e direitos reprodutivos, permitindo aos usuários trocar experiências e recorrer a analogias e seus contextos reais.

Cada usuário interessado pela esterilização cirúrgica participa uma única vez da atividade de educação em saúde durante o processo de aconselhamento contraceptivo.

O recrutamento dos participantes foi realizado no dia da atividade de educação em saúde pelos pesquisadores, os quais explicaram que participariam da atividade como observadores e ao final da mesma aplicariam um questionário para coleta de dados. Aqueles que aceitaram participar assinaram o Termo de Consentimento Livre e Esclarecido.

O questionário foi aplicado por meio de uma entrevista individual e em ambiente protegido, sendo composto por 14 questões fechadas e 06 questões abertas. 
As questões fechadas abordaram dados de caracterização sociodemográfica, antecedentes obstétricos e contraceptivos, enquanto as questões abertas se referiram à motivação para esterilização cirúrgica, dúvidas em relação aos métodos contraceptivos e procedimentos cirúrgicos, mudança de método contraceptivo e métodos que despertaram interesse após atividade de educação em saúde.

As respostas do questionário foram armazenadas em planilha do programa Microsoft Excel 2010 para quantificação dos dados. Para as respostas das questões fechadas foram calculadas frequências absolutas, relativas e médias. Para as respostas das questões abertas foram criadas quatro categorias, de acordo com os temas abstraídos (motivação para esterilização cirúrgica, dúvidas sobre os procedimentos de esterilização cirúrgica, métodos contraceptivos de interesse e mudança do método previamente escoIhido por outra opção contraceptiva), e calculadas as frequências absolutas e relativas. Os dados foram apresentados segundo análise descritiva por meio de tabelas.

\section{RESULTADOS}

Foram realizadas seis atividades de educação em saúde no período de setembro a dezembro de 2016 com participação de 46 pessoas. Somente uma delas não participou da pesquisa porque não pode aguardar o momento da entrevista ao final da atividade, caracterizando-se assim uma perda. O número máximo e mínimo de participantes foi de nove e cinco, respectivamente, com uma média de 7,7 participantes por atividade.

Dos 45 participantes, 26 eram mulheres (57,8\%) e 19 homens (42,2\%). A média de idade das mulheres e dos homens foi 31,4 e 37,5 anos, respectivamente. Outras caracteríticas sociodemograficas podem ser observadas na Tabela 1.

Tabela 1. Características sociodemográficas dos participantes das atividades de educação em saúde para esterilização cirúrgica. São Carlos/São Paulo, em 2016.

\begin{tabular}{|c|c|c|c|c|c|c|c|}
\hline \multirow{3}{*}{ Variáveis* $^{*}$} & \multirow{3}{*}{ Categoria } & \multicolumn{6}{|c|}{ Frequência } \\
\hline & & \multicolumn{2}{|c|}{ Mulheres } & \multicolumn{2}{|c|}{ Homens } & \multicolumn{2}{|c|}{ Total } \\
\hline & & $\mathrm{N}$ & $\%$ & $\mathrm{~N}$ & $\%$ & $\mathrm{~N}$ & $\%$ \\
\hline Gênero & & 26 & 57,8 & 19 & 42,2 & 45 & 100,0 \\
\hline Média de idade anos & & 31,4 & & 37,5 & & & \\
\hline \multirow{4}{*}{ Cor referida } & Branca & 12 & 46,2 & 09 & 47,4 & 21 & 46,7 \\
\hline & Parda & 07 & 26,9 & 07 & 36,8 & 14 & 31,1 \\
\hline & Preta & 06 & 23,1 & 03 & 15,8 & 09 & 20,0 \\
\hline & Amarela & 01 & 3,8 & 00 & 0,0 & 01 & 2,2 \\
\hline \multirow{3}{*}{ Estado civil } & Casados & 09 & 34,5 & 13 & 68,0 & 22 & 48,9 \\
\hline & Solteiros com união estável & 15 & 57,7 & 06 & 31,6 & 21 & 46,7 \\
\hline & Solteiros sem união estável & 02 & 7,8 & 00 & 0,0 & 02 & 4,4 \\
\hline \multirow{5}{*}{ Escolaridade } & Fund. incompleto & 05 & 19,2 & 02 & 10,5 & 07 & 15,6 \\
\hline & Fund. completo & 04 & 15,4 & 01 & 5,3 & 05 & 11,1 \\
\hline & Médio incompleto & 05 & 19,2 & 02 & 10,5 & 07 & 15,6 \\
\hline & Médio completo & 11 & 42,3 & 14 & 73,7 & 25 & 55,6 \\
\hline & Superior incompleto & 01 & 3,8 & 00 & 0,0 & 01 & 2,2 \\
\hline \multirow{4}{*}{ Religião } & Católico & 09 & 34,6 & 08 & 42,1 & 17 & 37,8 \\
\hline & Evangélico & 09 & 34,6 & 08 & 42,1 & 17 & 37,8 \\
\hline & Espírita & 00 & 0,0 & 02 & 10,5 & 02 & 4,4 \\
\hline & Outras & 08 & 30,8 & 01 & 5,3 & 09 & 20,0 \\
\hline
\end{tabular}

$\mathrm{N}$ : frequência absoluta; \%: frequência relativa; * $(n=45)$; Fund. = Fundamental. 
O número de filhos vivos entre os participantes variou de um a cinco, com predomínio de dois filhos $(51,1 \%)$. A maioria deles $(57,8 \%)$ tinha pelo menos dois filhos com os(as) atuais parceiros(as). O número de gestantes foi de $42,3 \%$ e entre elas cinco $(45,4 \%)$ tinham mais do que duas cesáreas anteriores (Tabela 2).

Tabela 2. Antecedentes obstétricos dos participantes das atividades de educação em saúde para esterilização cirúrgica. São Carlos/São Paulo, em 2016.

\begin{tabular}{|c|c|c|c|c|c|c|c|}
\hline \multirow{3}{*}{ Variáveis } & \multirow{3}{*}{ Categoria } & \multicolumn{6}{|c|}{ Frequência } \\
\hline & & \multicolumn{2}{|c|}{ Mulheres } & \multicolumn{2}{|c|}{ Homens } & \multicolumn{2}{|c|}{ Total } \\
\hline & & $\mathrm{N}$ & $\%$ & $\mathrm{~N}$ & $\%$ & $\mathrm{~N}$ & $\%$ \\
\hline \multirow{5}{*}{ No de filhos vivos* } & 1 & 02 & 7,8 & 00 & 0,0 & 02 & 4,4 \\
\hline & 2 & 11 & 42,3 & 12 & 63,1 & 23 & 51,1 \\
\hline & 3 & 09 & 34,6 & 05 & 26,3 & 14 & 31,1 \\
\hline & 4 & 03 & 11,5 & 01 & 5,3 & 04 & 9,0 \\
\hline & 5 & 01 & 3,8 & 01 & 5,3 & 02 & 4,4 \\
\hline \multirow{6}{*}{$\mathrm{N}^{\circ}$ de filhos vivos parceiro atual ${ }^{\star}$} & 0 & 02 & 7,8 & 00 & 0,0 & 02 & 4,4 \\
\hline & 1 & 05 & 19,2 & 01 & 5,3 & 06 & 13,3 \\
\hline & 2 & 13 & 50,0 & 13 & 68,4 & 26 & 57,8 \\
\hline & 3 & 03 & 11,5 & 04 & 21,0 & 07 & 15,6 \\
\hline & 4 & 03 & 11,5 & 00 & 0,0 & 03 & 6,7 \\
\hline & 5 & 00 & 0,0 & 01 & 5,3 & 01 & 2,2 \\
\hline \multirow{4}{*}{$\mathrm{N}^{\circ}$ de cesáreas de todas as mulheres ${ }^{\star *}$} & 0 & 10 & 38,6 & & & & \\
\hline & 1 & 08 & 30,7 & & & & \\
\hline & 2 & 05 & 19,2 & & & & \\
\hline & 3 & 03 & 11,5 & & & & \\
\hline \multirow{2}{*}{ Situação da mulher participante ${ }^{\star \star}$} & Gestante & 11 & 42,3 & & & & \\
\hline & Não Gestante & 15 & 57,7 & & & & \\
\hline
\end{tabular}

$\mathrm{N}$ : frequência absoluta; \%: frequência relativa; ${ }^{*}(n=45) ;{ }^{* *}(n=26)$.

A maioria dos participantes $(68,9 \%)$ estava em uso de algum método contraceptivo, sendo o mais citado (32,2\%) o contraceptivo hormonal oral combinado. O principal motivo de $11(78,6 \%)$ participantes não estarem usando método no momento se deveu a gestação em curso. Somente $4,4 \%$ dos participantes alegaram não terem usado algum método contraceptivo no passado (Tabela 3).

As respostas das questões abertas foram distribuídas em quatro categorias e quantificadas em frequência absoluta e relativa: motivação para esterilização cirúrgica, dúvidas sobre os procedimentos de esterilização cirúrgica, métodos contraceptivos de interesse e mudança do método previamente escolhido por outra opção contraceptiva.

Os principais motivos alegados pelos participantes para solicitar esterilização cirúrgica foram número suficiente de filhos $(42,2 \%)$, questões financeiras desfavoráveis $(33,3 \%)$, problemas de saúde da mulher $(9,0 \%)$ e outros motivos menos frequentes $(15,5 \%)$.

Quando os participantes foram entrevistados ao final da atividade de educação em saúde não acrescentaram nenhuma dúvida nova daquelas que já haviam sido problematizadas durante a mesma, sendo as principais sobre técnicas cirúrgicas da laqueadura tubária $(28,9 \%)$ e vasectomia $(22,2 \%)$, riscos da cirurgia propriamente dita $(44,4 \%)$ e da anestesia (33,3\%), além dos possíveis efeitos colaterais destes procedimentos, onde a impotência sexual $(52,6 \%)$ foi a maior preocupação entre os homens e a alteração do padrão menstrual $(46,1 \%)$ entre as mulheres. Os participantes tiveram mais de uma dúvida sobre o método escolhido que necessitou esclarecimentos. 
Tabela 3. Antecedentes do uso de métodos contraceptivos dos participantes das atividades de educação em saúde para esterilização cirúrgica. São Carlos/São Paulo, em 2016.

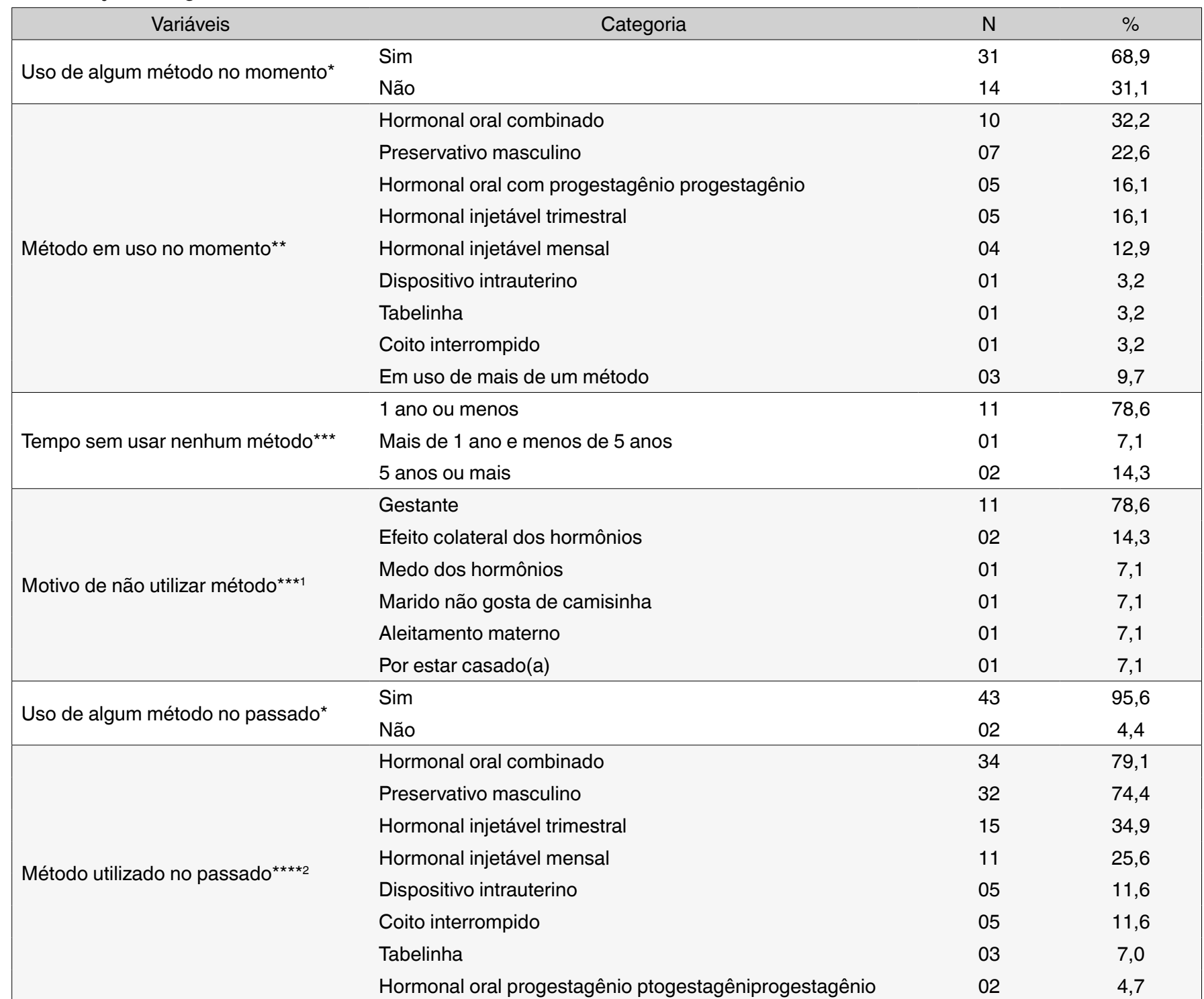

$\mathrm{N}$ : frequência absoluta; \%: frequência relativa; ${ }^{\star}(\mathrm{n}=45) ;{ }^{\star \star}(\mathrm{n}=31) ;{ }^{\star \star \star}(\mathrm{n}=14) ;{ }^{\star \star \star \star}(\mathrm{n}=43) ;{ }^{1}$ Citado mais de um motivo por pessoa; ${ }^{2}$ Citado mais de um método por pessoa.

Dois casais $(4,4 \%)$ mostraram-se interessados pela mudança da laqueadura tubária pela vasectomia após a atividade, porque pareceu ser um procedimento mais simples, rápido e com anestesia local. O interesse de mudança por outro método definitivo foi anotado em ficha de contrarreferência pela enfermeira para ser enviada aos profissionais das unidades de saúde de origem dos participantes, de forma que as devidas providências fossem tomadas. Os casais que mantiveram o desejo pelo procedimento inicialmente requerido alegaram estar satisfeitos com as informações recebidas e seguros quanto ao método definitivo que tinham escolhido, além de estarem cientes da dificuldade de irreversibilidade do mesmo.

O dispositivo intrauterino e o contraceptivo hormonal transdérmico foram os métodos reversíveis que chamaram a atenção de duas mulheres $(4,4 \%)$, porém elas disseram que a laqueadura tubária continuava ser o método escolhido. Nenhum participante manifestou o desejo de mudança do método definitivo por qualquer método reversível. 


\section{DISCUSSÃO}

Os achados desta pesquisa mostraram que as mulheres e os homens tinham idade acima de 30 anos quando iniciaram o processo de planejamento reprodutivo para esterilização cirúrgica, estando de acordo com a Lei da Esterilização Cirúrgica que prevê idade mínima acima de 25 anos. ${ }^{5}$

A idade média das mulheres (31,4 anos) e dos homens (37,5 anos) no início do processo do planejamento reprodutivo foi semelhante ao observado por Vieira e Souza, ${ }^{16}$ os quais relataram que a idade média dos indivíduos analisados foi de 34,4 anos, porém essa idade ocorreu no momento da esterilização cirúrgica e o tempo decorrido entre o início do processo e o procedimento foi em média de dois anos e meio.

Outros dados sociodemográficos dos participantes não divergiram muito dos encontrados por Vieira e Souza ${ }^{16}$ em que a maioria era de cor branca $(46,7 \% / 58,5 \%)$, casados $(48,9 \% / 58,5 \%)$ e evangélicos $(37,8 \% / 25,2 \%)$, respectivamente. Quanto ao nível de instrução na pesquisa em questão a maioria tinha ensino médio completo (55,6\%), enquanto no estudo de Vieira e Souza ${ }^{16}$ foi observado que $46 \%$ tinham de 5 a 8 anos de estudo, ou seja, no máximo ensino fundamental; semelhante ao estudo de Santos et al. ${ }^{17}$ que apontou que o ensino fundamental foi o nível de instrução mais frequente entre as mulheres que tinham realizado laqueadura tubária.

Diferente do estudo de Souza et al. ${ }^{15}$ em que $50 \%$ dos entrevistados responderam ter entre um e três filhos vivos, a maioria dos participantes $(82,2 \%)$ possuia entre dois e três filhos vivos, estando de acordo com a Lei da Esterilização Cirúrgica ${ }^{5}$ que prevê que este procedimento seja feito em pessoas com pelo menos dois filhos vivos.

As duas mulheres grávidas com um único filho desta pesquisa apresentavam situações que não as impediam de passar pelo processo de planejamento reprodutivo para esterilização cirúrgica, uma vez que uma delas tinha antecedente de trombose venosa profunda associada à pré-eclâmpsia na gravidez atual e queria que a laqueadura tubária fosse realizada na resolução da gestação; e a outra tinha uma cesárea anterior com um filho vivo e solicitava que o procedimento fosse feito logo após o parto.

A primeira situação está prevista na Lei da Esterilização Cirúrgica que define que a laqueadura tubária pode ser realizada na resolução da gestação quando a mulher apresentar cesarianas sucessivas anteriores ou quando for portadora de doença de base e a exposição a segundo ato cirúrgico ou anestésico representar maior risco à sua saúde; e a segunda situação pode ter o procedimento realizado, desde que aguardado até o $42^{\circ}$ dia pós-parto, confirmando dois filhos vivos. ${ }^{5,20}$

A mulher não ter filhos com o atual parceiro como foi observado nesta pesquisa não é um impediente para solicitação da esterilização cirúrgica, pois a lei prevê que o indivíduo que está requerendo o procedimento deve ter pelo menos dois filhos vivos. ${ }^{5}$ Assim, se ela já possui dois filhos vivos de outros relacionamentos está dentro dos critérios legais para o procedimento ser realizado.

No entanto, não significa que um indivíduo mesmo com número de filhos previstos pela lei não venha a se arrepender da esterilização cirúrgica no futuro como relatado por Carvalho et al., ${ }^{18}$ os quais verificaram que mais da metade das mulheres laqueadas que se arrependeram do procedimento possuía até dois filhos vivos. 
É preciso também estar atento à idade das mulheres, pois mesmo aquelas com mais de 25 anos podem se arrepender da esterilização cirúrgica como mencionado por Gossman et al.. ${ }^{21}$ estudo em que idade abaixo de 30 anos foi o mais forte preditor de arrependimento, chegando a $20,3 \%$ dos casos. Ou seja, entre 25 e 30 anos muitas mulheres podem se arrepender do procedimento mesmo estando com idade dentro dos critérios legais.

Estes dados são preocupantes e nos leva a pensar se algumas mulheres desta pesquisa não podem se arrepender no futuro, uma vez que a idade média foi de 31,4 anos, variando de 26 a 42 anos, e 42,3\% delas possuíam pelo menos dois filhos vivos.

Todavia, Ehman e Costescu ${ }^{22}$ relataram que independente da idade e do número de filhos a maioria das mulheres mesmo sendo informadas sobre o risco de arrependimento segue para o procedimento de esterilização cirúrgica. Os autores consideram que declinar de tal solicitação é uma forma de recusa consciente e esta não deve ser uma decisão clínica.

Entre as mulheres grávidas que manifestaram desejo pela laqueadura tubária $45,4 \%$ delas estava indo para a segunda ou terceira cesárea, caracterizando condição de exceção para esterilização cirúrgica no momento da resolução da gestação por cesáreas sucessivas. Uma delas tinha patologia com indicação médica para o procedimento e as demais tinham idade e número de filhos vivos previstos pela lei para que a laqueadura tubária pudesse ocorrer 42 dias após o parto. Todas estas condições estão previstas na Lei da Esterilização Cirúrgica. . $^{20}$

De acordo com Santos et al. ${ }^{17}$, as mulheres grávidas que frequentaram o ambulatório de gestação de risco e realizaram laqueadura tubária apresentaram $2,8 \%$ de arrependimento, levando os problemas de saúde explicar os baixos índices de arrependimento. Este achado pode ser tranquilizador em relação ao arrependimento das gestantes que manifestaram desejo pela laqueadura tubária na pesquisa em questão, uma vez que a maioria possuía indicação médica para o procedimento.

Os índices de uso de algum método contraceptivo nesta pesquisa $(68,9 \%)$ foram semelhantes aos observados na Pesquisa Nacional de Demografia e Saúde da Criança e da Mulher realizada em 2006, em mulheres entre 15 e 49 anos de idade $(67,8 \%)$. Também, foi condizente o achado em que o contraceptivo oral combinado, o preservativo masculino e o injetável mensal foram os métodos mais usados pelas muIheres. ${ }^{23}$ Os resultados desta pesquisa se aproximam dos dados encontrados no Plano Nacional de Saúde 2013 que apontam que $61,1 \%$ das mulheres sexualmente ativas entre 18 e 49 anos de idade utilizaram métodos contraceptivos nos últimos 12 meses para evitar gravidez. ${ }^{24}$

Entre os métodos contraceptivos utilizados no passado o contraceptivo oral combinado $(79,1 \%)$ e o preservativo masculino $(74,4 \%)$ foram os mais frequentes. Os índices elevados dos usuários desses métodos na solicitação pela esterilização cirúrgica podem suscitar vários tipos de especulações, entre eles que esses métodos não preveniram a gravidez como esperavam desejando um método mais seguro. Com índices inferiores, Souza et al. ${ }^{15}$ revelaram que embora $25 \%$ das mulheres tivessem usado contraceptivo oral combinado e $15 \%$ preservativo masculino com sucesso na prevenção da gravidez, ainda assim optaram pela laqueadura tubária. 
Deve-se ter cuidado acerca dos motivos que os indivíduos manifestam desejo pela esterilização cirúrgica, pois no estudo de Carvalho et al., ${ }^{18}$ os autores verificaram que o arrependimento pela laqueadura tubária foi maior entre as mulheres que mencionaram dificuldades financeiras e menor quando tinham muitos filhos ou problemas na gravidez, semelhante aos motivos referidos nesta pesquisa. Um dado importante que esses autores encontraram foi que o arrependimento pela laqueadura tubária entre as mulheres com um número ideal de filhos maior do que o número de filhos vivos foi quase 12 vezes mais, demonstrando que a avaliação prévia do número ideal de filhos pode ajudar na detecção de mulheres com maior risco para se arrepender deste procedimento, aspecto que não foi avaliado na pesquisa em questão.

Após a educação em saúde dois casais manifestaram interesse de mudança da laqueadura tubária para vasectomia, por ser um procedimento mais simples, rápido e de menor risco por usar anestesia local. Não foi possível confirmar se esta mudança de fato ocorreu, porém informações sobre o interesse dos casais foi relatada na contrarreferência para que os profissionais de saúde das unidades de origem dos participantes pudessem providenciar aconselhamentos com outros profissionais da equipe do planejamento reprodutivo.

O que vem sendo realizado está de acordo com a Lei da Esterilização Cirúrgica que prevê aconselhamento por equipe multiprofissional, propiciando que as pessoas ao conhecer os riscos, vantagens, desvantagens e eficácia dos métodos contraceptivos possam se conscientizar e refletir sobre o método definitivo escolhido, tendo possibilidade de mudança ao logo do processo, inclusive para métodos reversíveis, garantindo sua opção de escolha. ${ }^{5}$

O aconselhamento por meio da educação em saúde, utilizando a roda de conversa como abordagem, possibilita uma dinâmica proativa com expressão de sentimentos, dúvidas e questionamentos que estimulam a reflexão de valores e atitudes das pessoas, além de enfrentamento e superação das suas dificuldades. ${ }^{9,25}$ Além disso, a roda de conversa valoriza os conhecimentos e as experiências de cada participante, estimula o debate de opiniões sobre a temática proposta, bem como, permite a reflexão das manifestações apresentadas pelo grupo. ${ }^{26}$

Apesar, de poucos participantes terem manifestado o desejo de mudança de opção contraceptiva mesmo que definitiva, nos pareceu que a educação em saúde promoveu uma oportunidade de discussão sobre as experiências com os métodos utilizados e questionamentos sobre o método que estava sendo requerido, dando-nos a impressão que as atividades foram finalizadas satisfatoriamente.

Um ponto forte desta pesquisa foi o fato da quase totalidade dos indivíduos ter aceitado participar, sendo que somente uma pessoa não pode aguardar o momento da entrevista após o término da atividade de educação em saúde. Outro ponto forte foi a participação do(a) parceiro(a) quando o homem ou a mulher eram os usuários solicitantes pela esterilização cirúrgica. A participação do casal mostra a corresponsabilização pela escolha da esterilização cirúrgica, além de estarem juntos no aconselhamento se conscientizando e refletindo sobre uma possível mudança de método contraceptivo. ${ }^{16}$

A limitação se referiu a média de 7,7 participantes por atividade de educação em saúde, enquanto o número previsto era de 10, de modo que mais experiências poderiam contribuir para ampliar a conscientização da escolha. O fato da educação em saúde ser uma das primeiras etapas do processo de aconselhamento contraceptivo para esterilização cirúrgica, a avaliação das mudanças de opção do método inicialmente escolhido ficou um pouco limitada, mas foi suficiente para provocar reflexões sobre outras opções contraceptivas. 


\section{CONCLUSÕES}

O planejamento reprodutivo do município de São Carlos tem oferecido aconselhamento multiprofissional aos usuários que manifestam desejo pela esterilização cirúrgica, sendo a atividade de educação em saúde uma das etapas deste processo.

Constatamos que a educação em saúde possibilitou aos usuários conhecer mais sobre os métodos contraceptivos reversíveis e irreversíveis disponíveis, oportunizando vivenciar as experiências contraceptivas entre eles na roda de conversa. Apesar de alguns casais terem manifestado fazer vasectomia em vez de laqueadura tubária, não foi possível certificar se realmente o método inicialmente escolhido foi trocado. Mesmo algumas mulheres mostrando-se interessadas pelo dispositivo intrauterino e contraceptivo hormonal transdérmico disseram que a laqueadura tubária seria a melhor forma de contracepção.

Enfim, as atividades de educação em saúde permitiram aos usuários esclarecimentos de dúvidas sobre a esterilização cirúrgica e reflexão sobre a possibilidade de mudança do método inicialmente escolhido por outro irreversível ou mesmo reversível.

Esta pesquisa indica que estudos futuros com avaliação de todas as ações do aconselhamento contraceptivo para esterilização cirúrgica poderiam apresentar mais indícios sobre mudanças de opções para outros métodos contraceptivos.

\section{Contribuições dos autores:}

Concepção e/ou delineamento do estudo: EJF, CMS, FVRC, MC. Aquisição, análise ou interpretação dos dados: EJF, CMS, MC. Redação preliminar: EFF, MC. Revisão crítica da versão preliminar: EJF, FVRC, MC. Todos os autores aprovaram a versão final e concordaram em prestar contas sobre todos os aspectos do trabalho.

\section{REFERÊNCIAS}

1. Brasil. Ministério da Saúde. Assistência integral à saúde da mulher: bases de ação programática (Série B: Textos Básicos de Saúde, 6). Brasília (DF): Centro de Documentação do Ministério da Saúde; 1984; [acesso 2019 Jan 20]. Disponível em: http://bvsms.saude.gov.br/ bvs/publicacoes/assistencia_integral_saude_mulher.pdf

2. Negrão T. Uma ação sinérgica por direitos reprodutivos: uma história sem fim. Saúde Soc. 2012;21(Suppl 1):164-76.

3. Paiva CCN, Villar ASE, Souza MD, Lemos A. Educação em Saúde segundo os preceitos do Movimento Feminista: estratégias inovadoras para promoção da saúde sexual e reprodutiva. Esc Anna Nery. 2015 Out-Dez;19(4):685-91. DOI: https://doi.org/10.5935/1414-8145.20150092

4. Ventura M. Direitos reprodutivos no Brasil. $3^{\underline{a}}$ ed. Brasília (DF): Fundo de Populações das Nações Unidas (UNFPA); 2009.

5. Brasil. Lei $n^{\circ} 9.263$, de 12 de janeiro de 1996. Regula o $§ 7^{\circ}$ do art. 226 da Constituição Federal, que trata do planejamento familiar, estabelece penalidades e dá outras providências. Diário Oficial da União, Brasília (DF), 15 jan 1996; [acesso 2019 Jan 15]. Disponível em: http://www.planalto.gov.br/ccivil_03/LEIS/L9263.htm

6. Brasil. Ministério da Saúde. Secretaria de Atenção à Saúde. Departamento de Ações Programáticas Estratégicas. Política Nacional de atenção integral à saúde da mulher: princípios e diretrizes (Série C. Projetos, Programas e Relatórios). Brasília (DF): Ministério da Saúde; 2004; [acesso 2019 Jan 11]. Disponível em: http://bvsms.saude.gov.br/bvs/publicacoes/politica_nac_atencao_mulher.pdf

7. Brasil. Ministério da Saúde. Secretaria de Atenção à Saúde. Departamento de Ações Programáticas Estratégicas. Área Técnica de Saúde da Mulher. Direitos Sexuais e Direitos Reprodutivos: uma prioridade do governo (Série A. Normas e Manuais Técnicos) (Série Direitos Sexuais e Direitos Reprodutivos, Caderno no 1). Brasília (DF): Ministério da Saúde; 2005; [acesso 2019 jan 22]. Disponível em: http://bvsms.saude.gov.br/bvs/publicacoes/cartilha_direitos_sexuais_reprodutivos.pdf

8. Carvalho MLO, Schor N. Esterilização feminina: em busca do controle da própria fertilidade. Ciênc Cuid Saúde. 2012;11(5):95-101. DOI: https://doi.org/10.4025/cienccuidsaude.v11i5.17059 
9. Brasil. Ministério da Saúde. Secretaria de Atenção à Saúde. Departamento de Atenção Básica. Saúde sexual e saúde reprodutiva (Série

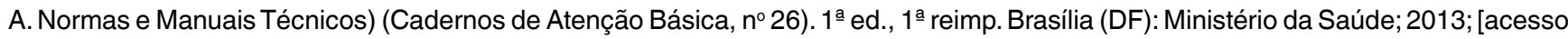
2019 Fev 14]. Disponível em: http://bvsms.saude.gov.br/bvs/publicacoes/saude_sexual_saude_reprodutiva.pdf

10. Silva KR, Santos AS, Pimenta DJ, Silva R, Lima MDO. Planejamento Familiar: importância das práticas educativas em saúde para jovens e adolescentes na Atenção Básica. Rev Gest Saúde. 2016;7(1):327-42.

11. Brasil. Ministério da Saúde. Secretaria de Atenção à Saúde. Departamento de Atenção Básica. Saúde na escola (Série B. Textos Básicos de Saúde) (Cadernos de Atenção Básica, n²2). Brasília (DF): Ministério da Saúde; 2009; [acesso 2019 Fev 16]. Disponível em: http://189.28.128.100/dab/docs/publicacoes/cadernos_ab/abcad24.pdf

12. Caetano AJ. Esterilização cirúrgica feminina no Brasil, 2000 a 2006. R. Bras. Est. Pop. 2014;31(2):309-31.

13. Brasil. Ministério da Saúde. Secretaria de Atenção a Saúde. Departamento de Ações Programáticas Estratégicas. Direitos sexuais, direitos reprodutivos e métodos anticoncepcionais (Série F. Comunicação e Educação em Saúde) (Direitos Sexuais e Direitos Reprodutivos, Caderno no 2). Brasília (DF): Ministério da Saúde; 2008; [acesso 2019 Jan 15]. Disponível em: https://www.nescon.medicina.ufmg.br/ biblioteca/registro/referencia/0000001546

14. World Health Organization (WHO), Department of Reproductive Health and Research (RHR), Johns Hopkins Bloomberg School of Public Health/Center for Communication Programs (CCP). Family Planning: A Global Handbook for Providers. $3^{\text {rd }}$ ed. Baltimore, Geneva: CCP, WHO; 2018.

15. Souza IBJ, Queiroz RCCS, Carvalho LKCA, Cunha CLF, Lago EC, Araújo TME. Ligadura tubária em mulheres de um município do interior do Maranhão. R Interd. 2013 Out-Dez;6(4):7-24.

16. Vieira EM, Souza L. A satisfação com o serviço de esterilização cirúrgica entre os usuários do Sistema Único de Saúde em um município paulista. Rev Bras Epidemiol. 2011;14(4):556-64.

17. Santos AM, Benute GRG, Nomura RMY, Miyadahira S, Lucia MCS, Francisco RPV. Arrependimento da realização da esterilização tubária em gestação de alto risco. Psicol Hosp. 2014;12(1):49-68.

18. Carvalho LEC, Cecatti JG, Osis MJD, Sousa MH. Número ideal de filhos e arrependimento pós-laqueadura. Rev Assoc Méd Bras. 2016 Out;52(5):293-7.

19. Araújo VS, Dias MD, Bustorff LACV. A instrumentalização da educação em saúde na atenção básica. Rev Enferm Ref. 2011;5:7-17. DOI: http://dx.doi.org/10.12707/RIII1143

20. Brasil. Ministério da Saúde. Secretaria da Assistência à Saúde. Portaria № 48, de 11 de fevereiro de 1999. Planejamento Familiar. Diário Oficial da União, Brasília (DF), 17 fev 1999; [acesso 2019 Fev 22]. Disponível em: http://bvsms.saude.gov.br/bvs/saudelegis/sas/1999/ prt0048_11_02_1999.html

21. Gossman W, Canela CD, Nama N. Tubal sterilization. StatPearls, Treasure Island: StatPearls Publishing; 2018.

22. Ehman D, Costescu D. Tubal sterilization in women under 30: case series and ethical implications. J Obstet Gynaecol Can. 2018;40(1):3640. DOI: https://doi.org/10.1016/j.jogc.2017.05.034

23. Brasil. Ministério da Saúde. PNDS 2006: Pesquisa Nacional de Demografia e Saúde da Mulher e da Criança. Brasília (DF): Ministério da Saúde; 2008; [acesso 2019 Fev 10]. Disponível em: http://bvsms.saude.gov.br/bvs/pnds/index.php

24. Instituto Brasileiro de Geografia e Estatística (IBGE). Pesquisa Nacional de Saúde 2013. Percepção do estado de saúde, estilos de vida e doenças crônicas. Rio de Janeiro (RJ): IBGE; 2014; [acesso 2019 Jan 21]. Disponível em: ftp://ftp.ibge.gov.br/PNS/2013/pns2013.pdf

25. Sampaio J, Santos GC, Agostini M, Salvador AS. Limits and potentialities of the circles of conversation: analysis of an experience with young people in the backcountry of Pernambuco, Brazil. Interface (Botucatu). 2014;18(Suppl 2):1299-1311. DOI: https://doi.org/10.1590/180757622013.0264

26. Dias ESM, Rodrigues ILA, Miranda HR, Corrêa JA. Roda de conversa como estratégia em saúde para a enfermagem. J Res: Fundam Care Online. 2018;10(2):379-84. DOI: http://dx.org/10.9789/2175-5361.rpcfo.v10.6053 


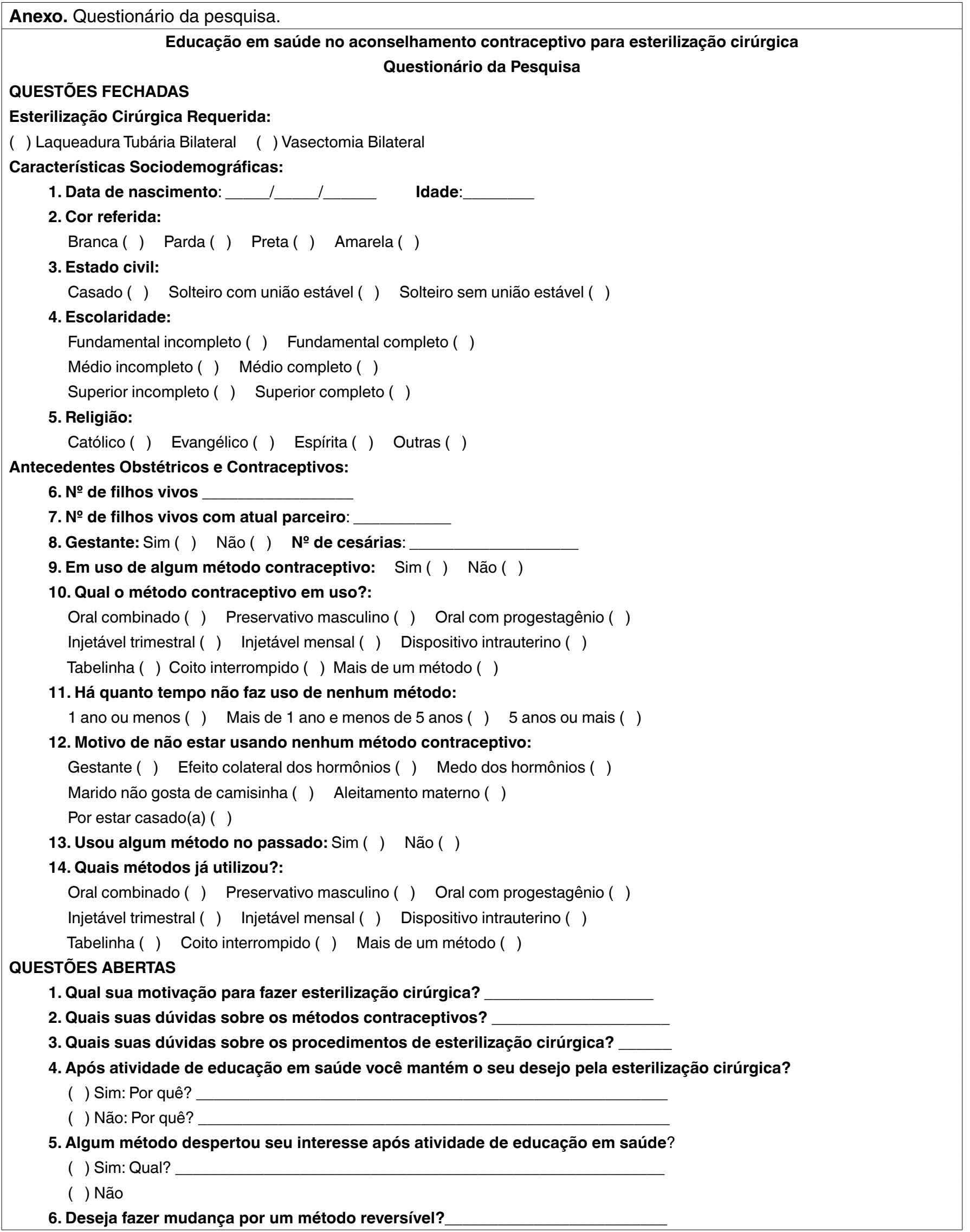

